\title{
Outcomes and Complications After Elbow Hemiarthroplasty for Complex Distal Humerus Fractures and Post Traumatic Arthritis. Review of the Literature
}

\section{Georgios Konstantopoulos ${ }^{1 *}$, Athanasios Serlis ${ }^{1}$, Panagiotis Poulios ${ }^{2}$ and Konstantinos Perpirakis ${ }^{3}$}

${ }^{1}$ Royal London Hospital, Department of Trauma and Orthopaedics, UK

${ }^{2}$ Barnet Hospital, Department of Trauma and Orthopaedics, UK

${ }^{3}$ Metropolitan General Hospital, Department of Trauma and Orthopaedics, Greece

*Corresponding Author: Georgios Konstantopoulos, Royal London Hospital,

Department of Trauma and Orthopaedics, UK.
Received: December 21, 2020

Published: January 16, 2020

(C) All rights are reserved by Georgios

Konstantopoulos., et al.

\section{Abstract}

Distal humerus fractures remain a challenge for the orthopaedic surgeon. In most of the cases the choice of treatment is surgical, and depending on the patient characteristics this would include ORIF, Total elbow replacement and elbow hemiarthroplasty. Although EHA is commonly used to deal with elbow fractures, to our knowledge there is one study that elbow hemiarthroplasty was used in young patients with post traumatic arthritis.

The aim of this article is to review the functional outcomes and the complications after elbow hemiarthroplasty for acute distal humerus fractures and treatment of post traumatic arthritis.

Keywords: Elbow Hemiarthroplasty; Outcomes; Complications; Post Traumatic Arthritis; Distal Humerus; Fracture

\section{Introduction}

Distal humerus fractures remain a challenge for the orthopaedic surgeon. They are rare injuries comprising $2-3 \%$ of all fractures and $30 \%$ of all elbow fractures [1].

There are contemporary techniques, allowing stable fixation of very complex injuries in the distal humerus, however there are fracture patterns that are not amenable to reconstruction due to the severity of the comminution, which makes the restoration of the articular surface very difficult or even impossible in some cases.

In most of the cases the choice of treatment is surgical, and depending on the patient characteristics this would include ORIF, Total elbow replacement (TER) and elbow hemiarthroplasty [2].
Although EHA is commonly used to deal with elbow fractures, to our knowledge there is one study that elbow hemiarthroplasty (EHA) was used in young patients with post traumatic arthritis.

The aim of this article is to review the outcomes and the complications after elbow hemiarthroplasty for acute distal humerus fractures and treatment of post traumatic arthritis.

\section{Methods}

The following terms were used in Pub Med search engine to identify relevant studies: elbow hemiarthroplasty, complications and outcomes, distal humerus, post traumatic arthritis. This search yielded 19 results. The bibliographies from these articles were scrutinised to identify any studies not caught in the search. Exclu- 
Outcomes and Complications After Elbow Hemiarthroplasty for Complex Distal Humerus Fractures and Post Traumatic Arthritis. Review of the Literature

sion criteria included Review papers, surgical techniques, cases that EHA was used other than trauma and post traumatic arthritis (rheumatoid arthritis, tumor, technique description article).

\section{Elbow hemiarthroplasty}

Historically, Mellen., et al. in 1947 were the first to describe four cases of EHA in 1947 using a non-anatomic acrylic implant that was used for salvage of high-energy injuries during the Second World War [3].

Street and Stevens in 1974 presented an arthroplasty of the distal humerus only, with a series of 9 patients, 3 with rheumatoid arthritis, 2 with ankylosing spondylitis and 5 with post traumatic arthritis.

In their series, the results were satisfactory in patients treated for post traumatic arthritis. Their complications included 2 loose implants, 1 case of HO, 1 case of ulnar neuropathy, 1 stiffness, 1 superficial infection [4].

Parsons., et al. in 2005 in a series of 8 patients reported in a mean 61months of follow up ROM 16-126 degrees, a mean ASES 81, 3 cases of prominent metalwork, ulnar neuropathy in 1 case and arthritis of the ulnohumeral joint in 1 case [5].

Adolfsson and his team according to their study in 2006 treated 4 patients with severely comminuted and irreparable distal humerus fractures. With a mean follow up of 10 months, 3 patients had an excellent outcome, and 1 good result, as shown by the MEPS. 1 complication was elbow weakness [6].

Hohman (2014), in a cohort of 8 patients with mean age 64 years old, out of which 7 were able to participate in the follow up process. They reported that the acute cases ( 5 patients) performed better than salvage cases ( 2 patients) on the Mayo Elbow Performance Score (80 points [range, 67-95 points] and 65 points [range, 50-80 points], respectively) and Disabilities of the Arm, Shoulder and Hand score (31 points [range, 2.5-68 points] and 39 points [range, 17-62 points], respectively). The mean arc of elbow flexion and extension was 96 degrees with mean flexion of 120 (range, and a mean extension loss of 19 . The mean arc of forearm rotation was 160degrees. Reoperation was deemed necessary in 4 patients due to painful retained hardware. In 5 cases ongoing post operative pain was reported [7].
Burkhart., et al. (2011) included in their study 10 female patients with a mean age of 75.2 years and a mean follow up 12.1 months who were treated with elbow HA either for osteoporotic, comminuted distal humerus fractures $(n=8)$ or for failed osteosynthesis of distal humerus fractures $(n=2)$. MEPS was good to excellent in 9 patients and fair in one. DASH was 11.5. Mean flexion was 124.5 degrees, pronation 80.5 degrees and supination was 79.5 degrees. One triceps weakness, one transient ulnar neuropathy, one superficial wound infection and 2 heteropic ossifications were seen. One patient developed $\mathrm{OA}$ in the proximal ulnar and radial articulation [8].

Argintar., et al. in 2012 studied retrospectively 10 patients who underwent elbow hemiarthroplasty and were followed up for 12 months mean time. Their mean MEPS were 77, DASH 13, mean ROM 22-121 Degrees. There was 1 case of ulnar neuritis and 1 case of prominent metalwork [9].

In a retrospective study Smith., et al. (2013) of 26 patients who were treated with EHA, reported good long term outcomes. Specifically, in 17 patients the ASES American shoulder and elbow score, Mayo elbow score, Quick DASH and EuroQol EQ5D showed good function and satisfaction. 4 patients had died, whereas 4 had been revised into a TER- 2 for periprosthetic fractures and 2 for loosening. The rest of the complications were ulnar neuropathy, stiffness, and wound necrosis. 13 patients had developed ulnar wear [10].

Nestorson., et al. (2015) reported reliable results in the medium term. In this study including 42 elderly patients with mean age 72 were followed up for a mean of 34.3 months. Functional outcome was measured with MEPS (90) and DASH (20). In terms of ROM the mean extension deficit was 23.5 degrees and mean flexion was 126.8 degrees. 4 patients had further surgical procedures for restricted ROM and one for partial instability. There was one case of loosening which was revised, 2 patients developed ulnar neuropathy, whereas radiographic signs of ulnar wear was found in 5 patients [11].

In 2015 Phadnis., et al. studied 16 patients with mean age 79 years old having undergone EHA for fracture, with a minimum 2 year follow up. Mean follow up was 35 months. MEPS was 89.6, Shortened DASH was 11.2 and Oxford Elbow Score 43.7. Mean flexion was 116 degrees, pro supination 172 degrees. There was radial head wear in 3 patients which was mild, whereas ulnar wear was 
Outcomes and Complications After Elbow Hemiarthroplasty for Complex Distal Humerus Fractures and Post Traumatic Arthritis. Review of the Literature

seen in 10 patients. There was 1 case of ulnar nerve neurapraxia which resolved [12].

In studying the midterm results of 6 patients having undergone EHA, Heijink., et al. in 2015 found that the implant survival was $100 \%$ after a mean 54 months follow up. Flexion -extension was 95.8 degrees, mean pro supination was 165 degrees. There were 3 cases of instability, 1 case of ulnar neuropathy. MEPS revealed 3 excellent results, 1 good and 1 poor. There was also one case of HO [13].

Lechasseur., et al. in 2015 published a case report with malunion of humeral trochlea fracture which was treated with an elbow hemiarthroplasty. In a follow up of 49 months, the ROM was 30-130 degrees, MEPS was 100, DASH was 8 . HO and prominent metalwork were also observed [14].

In 2016, Smith released their findings of a younger cohort of patients (mean age 44years) with EHA. 6 of them underwent the procedure and 2 of them were excluded as they had their elbows revised, due to aseptic loosening of the prosthesis. They reported satisfactory results using various scoring systems [15].

In a retrospective study Schultzel., et al. (2016), 10 patients underwent EHA by a single surgeon from 2012 to 2016. Patients were assessed for their ROM average flexion 128.7 degrees, extension deficit 27.1 degrees, 79.1 degrees of supination and 73.3 degrees of pronation -and their function was measured with MEPS 89.23, DASH, visual analog scale 2.43, Single Assessment Numeric Evaluation 74.14 and ASES 72.14. In terms of complications there was 1 fracture and one with symptoms of prominent hardware. 2 patients passed away and there was one who lost in follow up. Their mean age was 71.9 and the mean follow up 73.2 months [16].

In another study by Al-Hamdani., et al. (2019) 24 patients underwent EHA with acute communited distal humerus fracture during a 6 year period. The mean age was 65 years old and the median follow up 20 months.

Quantifying the outcomes with the Oxford elbow score, the results were good to excellent in 21 patients, fair in 2 and poor in 1 .
The Mayo Elbow performance score yieded good to excellent results in 19 patients, fair in 4 and poor in one patient. In their series the recorded 3 ulnar nerve neuropathies and 1 radiographic ulnar wear. None of them required revision surgery [17].

The largest study to our knowledge is registry based. Nestorson., et al. in 2019 included406 elbows(87 EHA) from 1999 and 2014 and a mean follow up 90 months for the Kudo implant and 48 for the Latitude elbow hemiarthroplasty, They reported 2 revisions in EHA in to TER, because of 1 loosening and one case of infection. There were 4 cases of HO, 3 fractures, 1 case of infection and 1 case of instability which they did not require revision or any other type of surgery [18].

Werthel., et al. (2019) studied the outcome of the EHA in post traumatic arthritis. They reported after a means 51 months follow up of 16 patients having undergone elbow hemiarthroplasty and using MEPs to assess the outcome,5 excellent results, 3 good results, 5 fair and one poor results. Furthermore, 5 had gone additional surgery and 2 were revised in TEA. In this study mean patient age was 45 years old. The complications that were reported included 2 ulnar fractures, 2 ulnar neuropathies, 1 broken metalwork, 1 septic loosening, 1 HO, 1 wound drainage and 1 seroma. There were 2 revisions into TEA, 1 due to ongoing pain and 1 due to septic loosening. ROM was 27-118 degrees [19].

\section{Discussion}

The studies included in this review show clearly that the early and midterm results and outcomes of patients undergoing elbow hemiarthroplasty are at the least satisfactory.

The commonest implant used is the Latitude system, seen in 13 studies. Other implants used include the Kudoand Sorbie Questor but less frequently.

The longest midterm mean follow up was 90 months [18].

The most recent study that has not been included in previous reviews is the one conducted by Werthel (2019). To our knowledge this is the only study that investigates the outcomes of EHA in patients with post traumatic arthritis [19]. 
Outcomes and Complications After Elbow Hemiarthroplasty for Complex Distal Humerus Fractures and Post Traumatic Arthritis. Review of the Literature

Most of the studies show similar outcome in ROM.

The most common complication in the mid term is UH wear.

In total 263 commercial implants for EHA from 2005 to 2019 the following complications were observed and reported:

The most common complication in the mid term is UH wear. The most commonly used functional score in the studies of this review was the MEPS. The lowest MEPS was recorded in the Argintar study in 2012, with a mean follow up of only 12 months. The highest score was achieved in the Phadnis study, with mean follow up of 35 months [9,12]. The functional outcome seems to be lower in the first year and it is improving in the midterm, but perhaps further studies are needed to support this hypothesis.

\begin{tabular}{|c|c|}
\hline Type of complication & N \\
\hline Infection & 8 \\
\hline Heterotopic ossification & 7 \\
\hline Instability & 4 \\
\hline Fracture & 6 \\
\hline UH wear & 40 \\
\hline Ulnar neuropathy & 19 \\
\hline Radiographic lucency/loosening & 15 \\
\hline Prominent metalwork & 22 \\
\hline Stiffness & 7 \\
\hline RH wear & 10 \\
\hline Triceps weakness & 3 \\
\hline Persistent pain & 1 \\
\hline Wound seroma & 1 \\
\hline Wound necrosis & 1 \\
\hline
\end{tabular}

Table 1: The reported complications in the literature.

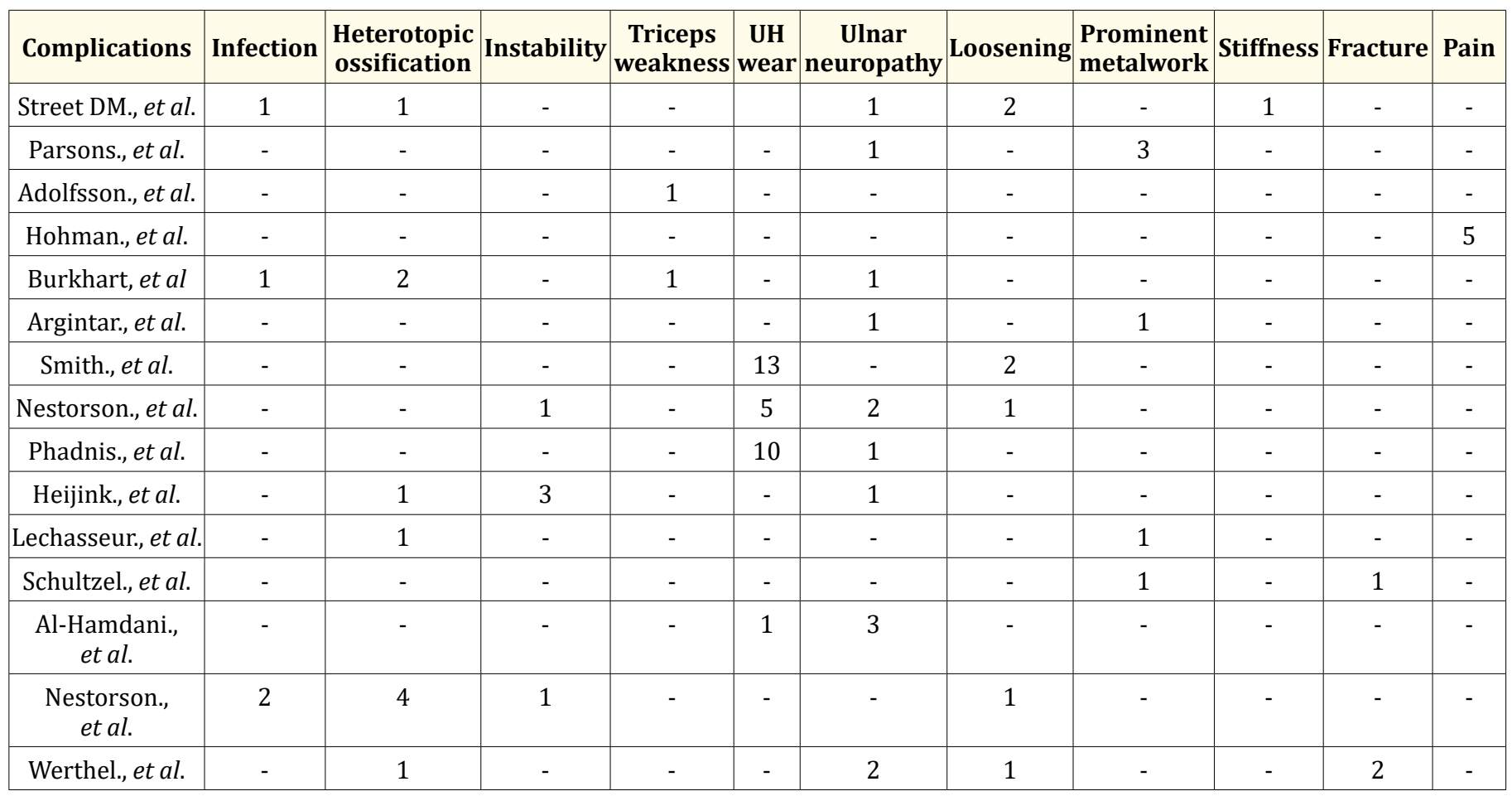

Table 2: The reported complications for the included studies. 
Outcomes and Complications After Elbow Hemiarthroplasty for Complex Distal Humerus Fractures and Post Traumatic Arthritis. Review of the Literature

\section{Conclusion}

EHA is a reliable method of treating distal humerus fractures, especially in the elderly population in the mid term, however more studies with longer follow up need to be conducted, extracting data from the national registries.

Additionally there is a need to define and compare results between ORIF and EHA, especially in elderly patients. This would be achieved potentially with a randomised control trial such as the one being conducted at the moment by Al Hamdani., et al. 44 patients will be randomised to either EHA or ORIF and they will be followed up at 3 months, 1, 2, 5 and 10 years [20].

Furthermore, it seems in the midterm it can provide with satisfactory results when treating post traumatic elbow arthritis in younger individuals, although more studies need to be conducted.

\section{Bibliography}

1. Rangarajan R and Papandrea RF. “DistalHumeral Hemiarthroplasty Versus Total Elbow Arthroplasty for Acute Distal Humeral Fractures". Orthopedics 40.1 (2017): 13-23.

2. ZalavrasCG and Papasoulis E. "Intra-articular fractures of the distal humerus- a review of the current practice". International Orthopaedics 42.11 (2018): 2653-2662.

3. Mellen RH and Phalen GS. "Arthroplasty of the elbow by replacement of the distal portion of the humerus with an acrylic prosthesis". Journal of Bone and Joint Surgery. American 29.2 (1947): 348-355.

4. Street DM and Stevens PS. "A humeral replacement prosthesis for the elbow: results in ten elbows". Journal of Bone and Joint Surgery. American 56.6 (1974): 1147-1158.

5. Parsons M., et al. "Elbow hemiarthroplasty for acute and salvage reconstruction of intra-articular distal humerus fractures". Techniques in Shoulder and Elbow Surgery 6.2 (2005): 87-97.

6. Adolfsson L and Hammer R. "Elbow hemiarthroplasty for acute reconstruction of intraarticular distal humerus fractures: a preliminary report involving 4 patients". Acta Orthopaedics 77.5 (2006): 785-787.
7. Hohman DW., et al. "Hemiarthroplasty of the distal humerus for acute and chronic complex intra-articular injuries". Journal of Shoulder and Elbow Surgery 23.2 (2014): 265-272.

8. Burkhart KJ., et al. "Distal humerus hemiarthroplasty of the elbow for comminuted distal humeral fractures in the elderly patient". Journal of Trauma 71.3 (2011): 635-642.

9. Argintar E., et al. "Hemiarthroplasty for the treatment of distal humerus fractures: short-term clinical results". Orthopedics 35 (2012): 1042-1045.

10. Smith GC and Hughes JSJ. "Unreconstructable acute distal humeral fractures and their sequelae treated with distal humeral hemiarthroplasty: a two-year to eleven-year follow-up". Shoulder Elbow Surgery 22.12 (2013): 1710-1723.

11. Nestorson J. et al. "Hemiarthroplasty for irreparable distal humeral fractures: medium-term follow-up of 42 patients". Bone Joint Journal 97-B (2015): 1377-1384.

12. Phadnis J., et al. "Elbow hemiarthroplasty using a "triceps-on" approach for the management of acute distal humeral fractures". Journal of Shoulder and Elbow Surgery 24.8 (2015): 1178-1186.

13. Heijink A. "Distal humerus prosthetic hemiarthroplasty: midterm results". Strategies in Trauma and Limb Reconstruction 10 (2015): 101-108.

14. Lechasseur B., et al. "Incipient malunion of an isolated humeral trochlea fracture treated with an elbow hemiarthroplasty: case report". Journal of Hand Surgery (American Volume) 40.2 (2015): 271-275.

15. Smith GC., et al. "The clinical outcome and activity levels of patients under 55 years treated with distal humeral hemiarthroplasty for distal humeral fractures: minimum 2-year followup". Shoulder Elbow 8.4 (2016): 264-270.

16. Schultzel M., et al. "Hemiarthroplasty for the treatment of distal humeral fractures: midterm clinical results". Journal of Shoulder and Elbow Surgery 26.3 (2017): 389-393. 
17. Al-Hamdani A., et al. "Good outcome after elbow hemiarthroplasty in active patients with an acute intra-articular distal humeral fracture". Shoulder Elbow Surgery 28.5 (2019): 925930.

18. Nestorson J., et al. "Arthroplasty as primary treatment for distal humeral fractures produces reliable results with regards to revisions and adverse events: a registry based study". Journal of Shoulder and Elbow Surgery 28.4 (2019): e104-110.

19. Werthel JD., et al. "Outcomes After Hemiarthroplasty of the Elbow for the Management of Posttraumatic Arthritis: Minimum 2-Year Follow-up". American Academy of Orthopaedic Surgeons 27.19 (2019): 727-735.

20. Ali Al-Hamdani A., et al. "Elbow hemiarthroplasty versus open reduction and internal fixation for A0/OTA type 13 C2 and C3 fractures of distal humerus in patients aged 50 years or above: a randomized controlled trial. -you can write it in the discussionsession-the need to compare the ORIF to EHA". Trials 21 (2020): 497.

\section{Assets from publication with us}

- Prompt Acknowledgement after receiving the article

- Thorough Double blinded peer review

- Rapid Publication

- Issue of Publication Certificate

- High visibility of your Published work

Website: https://www.actascientific.com/ Submit Article: https://www.actascientific.com/submission.php Email us: editor@actascientific.com

Contact us: +919182824667

Citation: Georgios Konstantopoulos., et al. "Outcomes and Complications After Elbow Hemiarthroplasty for Complex Distal Humerus Fractures and Post Traumatic Arthritis. Review of the Literature". Acta Scientific Orthopaedics 4.2 (2021): 21-26. 